\title{
A Distinguishing Attack of SNOW 2.0 with Linear Masking Method
}

\author{
Dai Watanabe $^{1}$, Alex Biryukov ${ }^{2}$, and Christophe De Cannière ${ }^{2}$ \\ 1 Systems Development Laboratory, Hitachi, Ltd., \\ 292 Yoshida-cho, Totsuka-ku, Yokohama, 244-0817, Japan \\ daidai@sdl.hitachi.co.jp \\ 2 Katholieke Universiteit Leuven, Dept. Electrical Engineering-ESAT, \\ Kasteelpark Arenberg 10, B-3001 Heverlee, Belgium \\ \{Alex.Biryukov, Christophe.DeCanniere\}@esat.kuleuven.ac.be
}

\begin{abstract}
SNOW 2.0 was developed by Johansson and Ekdahl in 2002, as a modified version of SNOW 1.0. In this paper we present the application of linear (masking) attack to SNOW 2.0 stream cipher. Our attack requires $2^{225}$ output words $\left(2^{230}\right.$ bits $)$ and $2^{225}$ steps of analysis to distinguish the output of SNOW 2.0 from a truly random bit sequence.
\end{abstract}

Keywords: Stream cipher, SNOW, Linear cryptanalysis

\section{Introduction}

A keystream generator (KSG) is a cryptographic primitive used for data encryption and random number generation. Many KSGs have been proposed so far. Most of them adopted bit-wise operations and used linear feedback shift registers (LFSRs). These KSGs are hardware oriented designs so that the cost (required gate size for the implementations) is quite small compared to most of block ciphers these days. In addition, the security evaluation for this class of KSGs is a well-studied problem. The output of the LFSRs has good statistical properties, e.g., long period, unbiased bit frequency, and so on. Only the weakness of the output of the LFSRs is the linear property derived from the update function and a lot of works relevant to the weakness have been presented [Ru86].

On the other hand, progress in the chip manufacturing technology relaxes the limitation of the gate count. In addition, the increase of cryptographic applications in software motivates recent KSG designs, for example SEAL [RC94] [RC98], PANAma [DC98], SOBER [Ro98], MUGI [WFYP02], SNOW [EJ00] [EJ02b], Scream [CHJ02a], etc. The size of their internal state increases, and they adopt block-wise (e.g. 8-bit or 32-bit) operations for running fast on today's 8-bit- or 32-bit-processors. Some of them still adopt LFSRs as a primitive of their algorithms because of the well studied properties of the LFSRs.

SNOW family is a typical example of this class of KSGs. SNOW 1.0 is an LFSR-based stream cipher proposed by Johansson and Ekdahl [EJ00] and the 
key length is 128 or 256 bits. The significant property is the feedback polynomial defined over $\mathrm{GF}\left(2^{32}\right)$ and it enables SNOW 1.0 to run fast especially on 32bit processors. SNOW was submitted to NESSIE (New European Schemes for Signatures, Integrity, and Encryption ) project in 2000.

NESSIE project requires that a stream cipher provides full security, i.e., no security flaw can be found with calculation complexity less than exhaustive key search. We follow the style because the key length is often identified by the security of a cipher in real use, if no security bound is clearly defined.

SNOW 1.0 is designed to be secure as a 256-bit key cipher, but two different attacks have been presented. One is Guess-and-Determine attack by Hawkes and Rose [HR02]. This is a key recovery attack and the complexity of the attack is $O\left(2^{224}\right)$. Another attack is a distinguishing attack by Coppersmith et al. [CHJ02b]. The complexity of this attack is about $2^{100}$. The basic idea of this attack, developed by Golic [Go96], applies the technique of linear cryptanalysis on block ciphers [Ma94] for distinguishing the outputs of a KSG from a truly random bit sequence. This attack has been applied to RC4 [Go97], SOBER-t16 and t32 [EJ02a] so far. The attack of Coppersmith is the variant and is especially efficient for the cipher that consists of LFSRs with memory.

SNOW 2.0 is an improved version of SNOW 1.0 aimed to be secure against these attacks and remain to be efficient both in software and hardware implementations. However, no security evaluation against these attacks have been presented in the past proposal papers. In this paper we develop the distinguishing attack on SNOW 2.0 by linear masking method. This attack uses $2^{230}$ bits of the stream and $2^{225}$ steps of analysis, which is faster than exhaustive search for the 256-bit key. This result shows that though SNOW 2.0 has improved security against linear masking compared to SNOW 1.0 it is still vulnerable to this attack.

The rest of this paper is organized as follows. Firstly we briefly describe the algorithm of SNOW 1.0 in Sect. 2. Then we introduce linear masking method and show its application to SNOW 1.0 in Sect. 3. We describe the algorithm of SNOW 2.0 in Sect. 4 and show how to apply linear masking method to SNOW 2.0 in Sect. 5. At last we summarize the result in Sect. 6.

\section{The Algorithm of SNOW 1.0}

In this section, we present a brief description of the algorithm of SNOW 1.0. We ignore the key setup process because our attack is independent of this process.

SNOW 1.0 is a stream cipher which has a 128- or a 256-bit secret key and a 128-bit initial vector as inputs. It is based on LFSR over $\operatorname{GF}\left(2^{32}\right)$ and the feedback polynomial is given by

$$
p(x)=x^{16}+x^{13}+x^{7}+\alpha^{-1},
$$

where $\alpha$ is a root of the polynomial $y^{32}+y^{29}+y^{20}+y^{15}+y^{10}+y+1 \in \operatorname{GF}(2)[y]$.

The state of LFSR is denoted by $\left(s_{t+16}, s_{t+15}, \ldots, s_{t+1}\right)$. Each 32-bit word $s_{t+i}$ is an element of $\mathrm{GF}\left(2^{32}\right)$. 


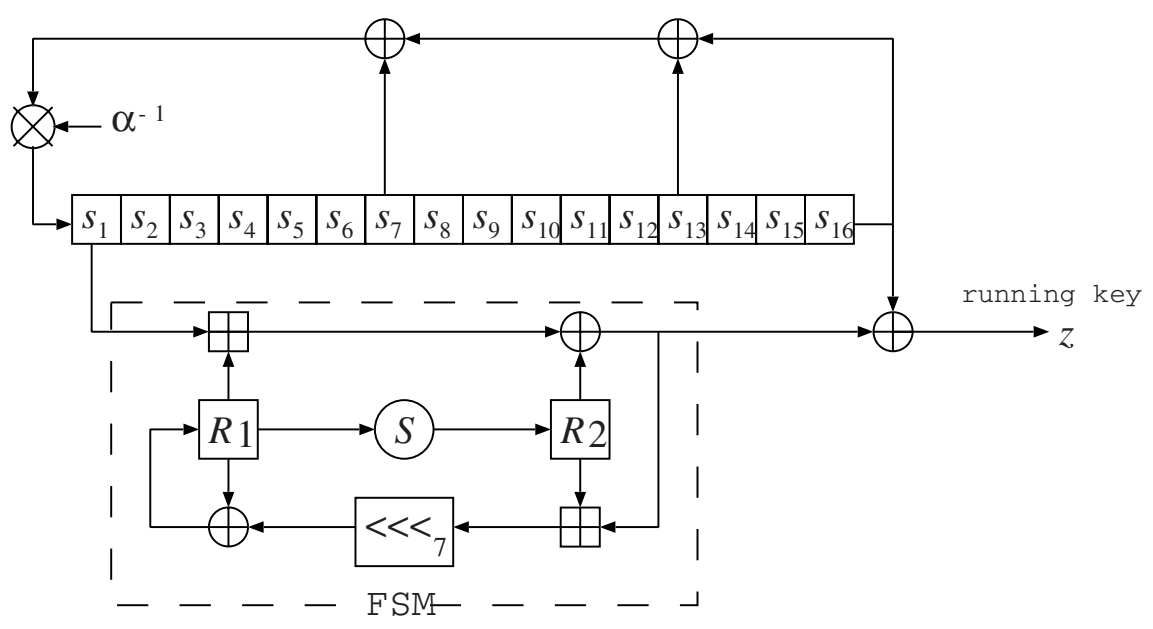

Fig. 1. The structure of SNOW 1.0

The Finite State Machine (FSM) is an additional internal state consisting of two 32-bit registers $R 1$ and $R 2$. The subscript $t$ is used to specify the time, e.g. $R 1_{t}, R 2_{t}$. The output of the FSM $F_{t}$ is given by

$$
F_{t}=\left(s_{t+1} \boxplus R 1_{t}\right) \oplus R 2_{t}, \quad t \geq 0,
$$

where $\boxplus$ is the addition modulo $2^{32}$ and $\oplus$ is the XORing operation. The keystream $z_{t}$ is given by

$$
z_{t}=F_{t} \oplus s_{t+16}, \quad t \geq 1
$$

Two registers $R 1$ and $R 2$ are updated as follows:

$$
\begin{aligned}
& \left.R 1_{t+1}=\left(F_{t} \boxplus R 2_{t}\right) \ggg 7\right) \oplus R 1_{t}, \\
& R 2_{t+1}=S\left(R 1_{t}\right) .
\end{aligned}
$$

The 32-bit non-linear transformation $S$ consists of four identical 8-to- 8 bit S-boxes and a permutation of the resulting bits. See [EJ00] for more detail.

\section{$3 \quad$ Linear Masking Method}

Distinguishing attack is a generic approach which allows to distinguish the output of the target KSG from a truly random sequence of the same length. The distinguishing method (or algorithm) is called a distinguisher. In this section, we explain the basic idea of linear masking method, which is a distinguishing attack proposed by Coppersmith et al. and show the application to SNOW 1.0. 


\subsection{General Description}

Linear masking method [CHJ02b] is a general attack against a large class of KSGs. The basic idea of the attack is the same as linear cryptanalysis against block ciphers proposed by Matsui [Ma94].

The first step of linear cryptanalysis is to select several bit of inputs, outputs, and a fixed secret key. The sum of the selected bits is called a linear approximation. Then the attacker calculates the bias of selected bits through amount of known plaintexts. The target encryption function can be distinguished from a random permutation with this distinguisher if the bias is sufficiently large. Furthermore, the attacker can conjects the sum of key bits from the bias by maximum likelihood method. The main interest in the study of linear cryptanalysis is how to find the best approximation, which is the linear approximation with largest bias. Matsui shows how to construct the linear approximation in general by the iterative structure of block ciphers. His construction firstly searches for the best approximation of the round function of the target block cipher, and combines them to remove the terms of intermediate bits.

Linear masking method pays attention to the bias of the linear approximation consisting of only output bits and distinguishes the output of the target KSG from a truly random bit sequence. This attack is applicable if the internal state of the target KSG can be divided into the linear (or low-diffusion) part and non-linear part. The basic procedure of linear masking method is as follows:

Step 1. Divide the algorithm of a keystream generator into two parts; the linear part and the non-linear part. Let us denote the linear relation of the linear part by

$$
\varphi(s, t)=\bigoplus_{j} c_{j} s_{t+j}=0, \quad c_{j} \in \mathrm{GF}(2),
$$

where $\left\{s_{t+j}\right\}_{j}$ is the internal state of the linear part.

Step 2. Find the best approximation of the non-linear part:

$$
\bigoplus_{i} \Gamma_{i} s_{t+i}=\bigoplus_{k} \Gamma_{k}^{\prime} z_{t+k}
$$

where $\left\{z_{t+k}\right\}_{k}$ is the output blocks. 32-bit values $\left\{\Gamma_{i}\right\}_{i}$ and $\left\{\Gamma_{k}^{\prime}\right\}_{k}$ are called masks and $\Gamma_{i} s_{t+i}$ is the inner product of $\Gamma_{i}$ and $s_{t+i}$ over GF(2). Denote the probability that this equation holds by $p$, and define the bias $\epsilon_{p}$ by $p=1 / 2+\epsilon_{p}$. Let us denote the left hand of the equation by $f(s, t)$ and the right hand by $g(z, t)$ respectively. If the equation holds with the bias $\epsilon$, about $\epsilon^{2}$ rounds outputs is needed to distinguish the outputs from a truly random bit sequence.

Step 3. Construct the linear approximation consisting of only outputs by combining above two equations 2 and 3 , and calculate the bias via piling up lemma [Ma94]:

$$
0=\bigoplus_{i} \Gamma_{i} \varphi(s, t+i)=\bigoplus_{j} c_{j} f(s, t+j)=\bigoplus_{j} c_{j} g(z, t+j) .
$$




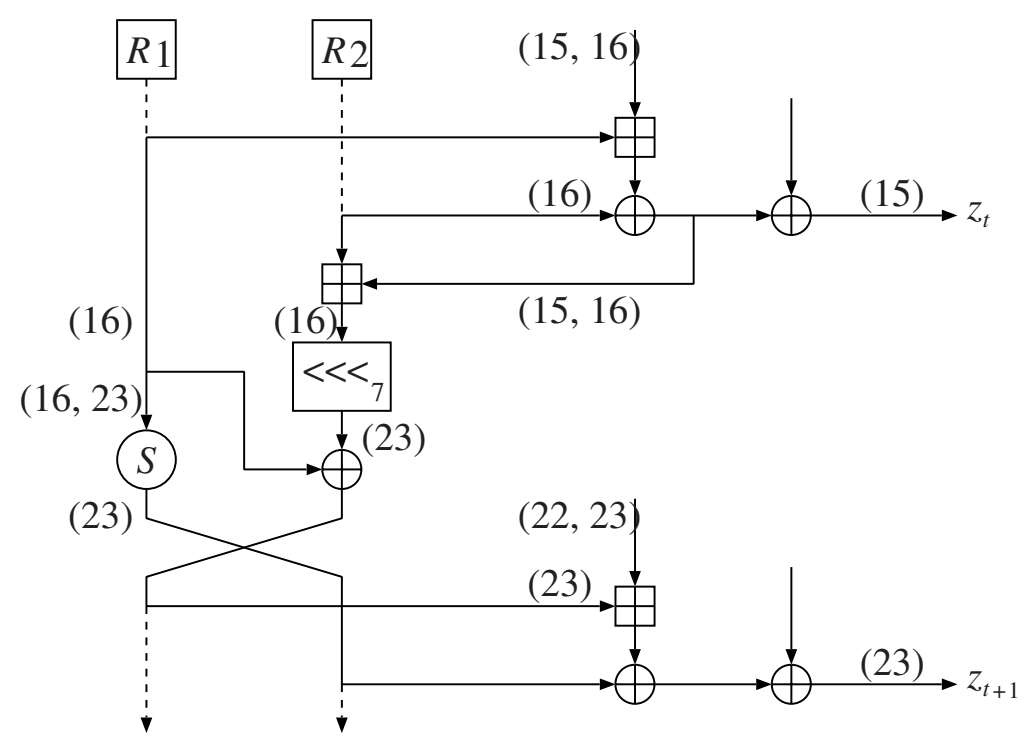

Fig. 2. The linear approximation of SNOW 1.0

\subsection{Application to SNOW 1.0}

The expression over $\mathrm{GF}(2)$ of the feedback polynomial yields a linear relation. But it has many coefficients so that linear masking method is not efficient. In [CHJ02b] Coppersmith et al. pointed out that the feedback polynomial has only one coefficient $\alpha$ which does not belong to GF(2). They used Frobenius map to eliminate $\alpha$ and constructed a linear relation over $\mathrm{GF}(2)$ with 6 terms. I.e., the polynomial

$$
p(x)^{2^{32}}+p(x)=x^{16 \times 2^{32}}+x^{13 \times 2^{32}}+x^{7 \times 2^{32}}+x^{16}+x^{13}+x^{7}
$$

gives the linear relation. On the other hand, the linear approximation of nonlinear part is given by the following 2-rounds approximation of FSM (See Fig. 2):

$$
\left(s_{t+1}\right)_{15,16} \oplus\left(s_{t+16}\right)_{15} \oplus\left(s_{t+15}\right)_{23} \oplus\left(s_{t+17}\right)_{22,23}=\left(F_{t}\right)_{15} \oplus\left(F_{t+1}\right)_{23} .
$$

They declared that the bias of the approximation is at least $2^{-9.3}$. Hence, the linear approximation derived from Eq. 5, 6 is $2^{5} \cdot 2^{-9.3 \times 6}=2^{-50.8}$ and $2^{101.6}$ rounds outputs is needed to distinguish the output of SNOW 1.0 from a truly random bit sequence.

\section{The Algorithm of SNOW 2.0}

In this section, we present a brief description of the algorithm of SNOW 2.0 except for the key setup process. 


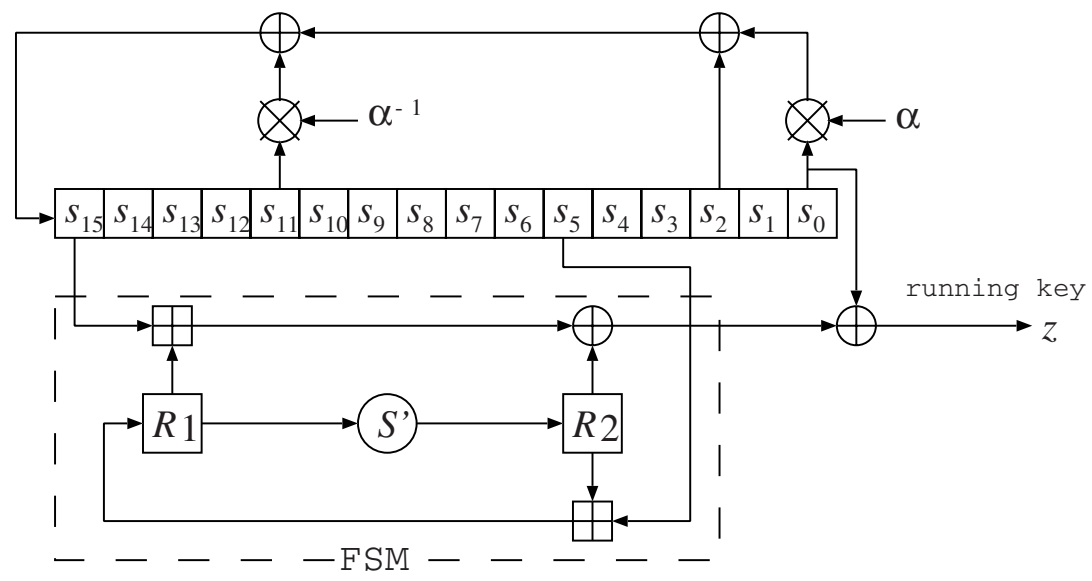

Fig. 3. The structure of SNOW 2.0

SNOW 2.0 is a stream cipher which has a 128- or a 256-bit secret key and a 128-bit initial vector. It is based on LFSR over $\operatorname{GF}\left(2^{32}\right)$ and the feedback polynomial is given by

$$
\pi(x)=\alpha x^{16}+x^{14}+\alpha^{-1} x^{5}+1,
$$

where $\alpha$ is a root of the polynomial $y^{4}+\beta^{23} y^{3}+\beta^{245} y^{2}+\beta^{48} y+\beta^{239} \in \operatorname{GF}\left(2^{8}\right)[y]$, and $\beta$ is a root of $z^{8}+z^{7}+z^{5}+z^{3}+1 \in \mathrm{GF}(2)[z]$.

The state of LFSR is denoted by $\left(s_{t+15}, s_{t+14}, \ldots, s_{t}\right)$. Each $s_{t+i}$ is an element of $\operatorname{GF}\left(2^{32}\right)$. Note that the sequence is opposite to that of SNOW 1.0 and the index starts at zero.

The Finite State Machine (FSM) has two 32-bit registers, $R 1$ and $R 2$. The output of the FSM $F_{t}$ is given by

$$
F_{t}=\left(s_{t+15} \boxplus R 1_{t}\right) \oplus R 2_{t}, \quad t \geq 0,
$$

and the keystream $z_{t}$ is given by

$$
z_{t}=F_{t} \oplus s_{t}, \quad t \geq 1
$$

Two registers $R 1$ and $R 2$ are updated as follows:

$$
\begin{aligned}
& R 1_{t+1}=s_{t+5} \boxplus R 2_{t}, \\
& R 2_{t+2}=S^{\prime}\left(R 1_{t}\right) .
\end{aligned}
$$

$S^{\prime}$ is the one column of AES, i.e. the combination of S-box layer (substitution byte) and the linear diffusion layer (mix column). The S-box and the matrix for the linear diffusion are denoted by $S_{R}$ and $M$ respectively. 


\section{The Attack on SNOW 2.0}

\subsection{The Basic Idea}

The difficulty in applying linear masking method to SNOW family is caused by the multiplications over $\mathrm{GF}\left(2^{32}\right)$ in the LFSRs. Considering that the feedback polynomial is defined over $\mathrm{GF}(2)$ make it easy to apply linear masking method, however the number of terms of the polynomial becomes large. Hence the bias of whole linear approximation becomes small even though the bias of Eq. 3 is large so that the attack is no longer effective.

In the case of SNOW 1.0, Coppersmith et. al. noticed that only one coefficient of the feedback polynomial is not equal to 1 and use Frobenius map to remove this coefficient to construct another linear relation without the multiplication with the element of $\mathrm{GF}\left(2^{32}\right)$. On the other hand, the feedback polynomial of SNOW 2.0 has two different coefficients which belong to GF $\left(2^{32}\right)$. This improvement of the feedback polynomial makes it difficult to apply the technique to SNOW 2.0 which is used against SNOW 1.0.

In the following, we examine to apply the feedback polynomial itself as the linear equation $\varphi$ to combine the plural approximations of FSM. Assume that the linear approximation of FSM is given by

$$
\bigoplus_{i} \Gamma_{i} s_{t+i} \oplus \bigoplus_{j} \Gamma_{j}^{\prime} z_{t+j}=0 .
$$

On the other hand, the internal state of LFSR $s$ at time $t$ satisfies the following linear relation for any mask $\Gamma$ :

$$
\Gamma\left(s_{t+16} \oplus \alpha^{-1} \cdot s_{t+11} \oplus s_{t+2} \oplus \alpha \cdot s_{t}\right)=0 .
$$

Multiplying the element of the finite field is a linear transformation over GF(2), so that this equation can be re-written as follows:

$$
\Gamma s_{t+16} \oplus\left(\Gamma \cdot \alpha^{-1}\right) s_{t+11} \oplus \Gamma s_{t+2} \oplus(\Gamma \cdot \alpha) s_{t}=0,
$$

where the multiplications $\Gamma \cdot \alpha$ and $\Gamma \cdot \alpha^{-1}$ are the composition of two linear transformation over $\mathrm{GF}(2)$.

Hence if Eq. 8 holds for the mask sets $\left\{\Gamma_{i}\right\}_{i},\left\{\Gamma_{i} \cdot \alpha\right\}_{i}$, and $\left\{\Gamma_{i} \cdot \alpha^{-1}\right\}_{i}$ with a large bias, the attacker can construct the linear approximation consisting of only output bits with large bias from these linear approximations of FSM and the linear relation Eq. 10.

\subsection{The Linear Approximation of FSM}

Figure 4 shows the 2-rounds approximation of FSM:

$$
\Gamma_{0} s_{t} \oplus \Gamma_{1} s_{t+1} \oplus \Gamma_{5} s_{t+5} \oplus \Gamma_{15} s_{t+15} \oplus \Gamma_{16} s_{t+16}=\Gamma_{0} z_{t} \oplus \Gamma_{1} z_{t+1} .
$$

In this subsection, we calculate the bias of Eq. 11 . 


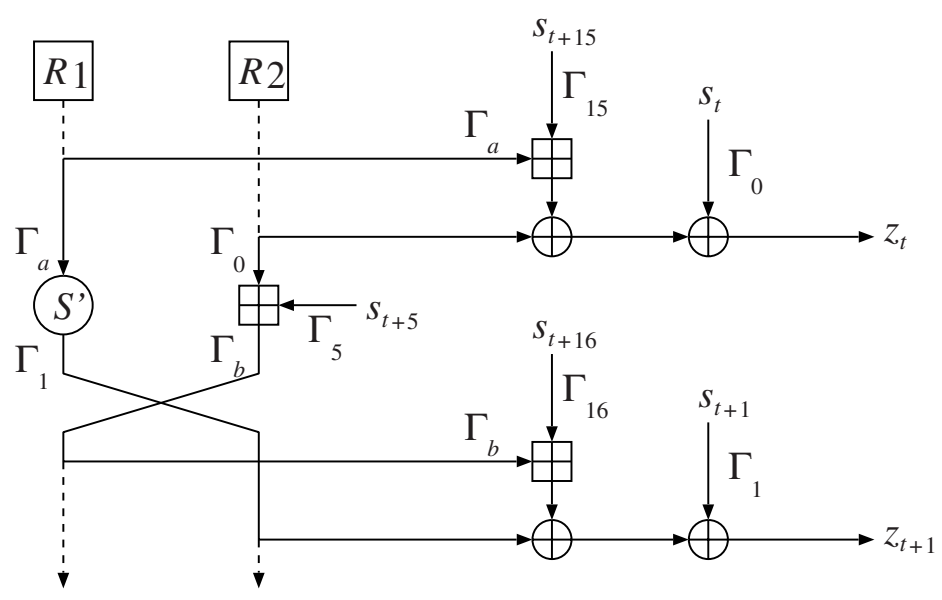

Fig. 4. The linear approximation of FSM

There are two non-linear operations in the linear approximation of FSM (11): one is the 32-bit permutation $S^{\prime}$ and the other is the additions modulo $2^{32}$ used three times. To simplify the search for the mask sets, we assume that the values of the masks do not change at three addition operands, i.e. we assume that the approximation $\Gamma x \oplus \Gamma y=\Gamma(x \boxplus y)$ has a large bias. In fact, this assumption holds in many cases.

Under the assumption, all masks $\Gamma_{i}$ in the linear approximation (11) must have the same value. In the following, we ignore the subscripts and denote these masks as $\Gamma$. The linear approximation consisting of only outputs can be constructed from two equations Eq. 10 and 11:

$$
\begin{aligned}
0= & \Gamma\left(s_{t+16} \oplus \alpha^{-1} \cdot s_{t+11} \oplus s_{t+2} \oplus \alpha \cdot s_{t}\right) \\
& \oplus \Gamma\left(s_{t+17} \oplus \alpha^{-1} \cdot s_{t+12} \oplus s_{t+3} \oplus \alpha \cdot s_{t+1}\right) \\
& \oplus \Gamma\left(s_{t+21} \oplus \alpha^{-1} \cdot s_{t+16} \oplus s_{t+7} \oplus \alpha \cdot s_{t+5}\right) \\
& \oplus \Gamma\left(s_{t+31} \oplus \alpha^{-1} \cdot s_{t+26} \oplus s_{t+17} \oplus \alpha \cdot s_{t+15}\right) \\
& \oplus \Gamma\left(s_{t+32} \oplus \alpha^{-1} \cdot s_{t+27} \oplus s_{t+18} \oplus \alpha \cdot s_{t+16}\right) \\
= & \Gamma\left(z_{t+2} \oplus z_{t+3} \oplus z_{t+16} \oplus z_{t+17}\right) \\
& \oplus\left(\Gamma \alpha^{-1}\right)\left(z_{t+11} \oplus z_{t+12}\right) \oplus(\Gamma \alpha)\left(z_{t} \oplus z_{t+1}\right) .
\end{aligned}
$$

The search for the linear approximation of FSM is reduced to search the following two approximations:

$$
\begin{aligned}
\Gamma S^{\prime}(x) \oplus \Gamma x & =0, \\
\Gamma x \oplus \Gamma y & =\Gamma(x \boxplus y) .
\end{aligned}
$$

Let us denote the biases of Eq. 13 and 14 as $\epsilon_{S^{\prime}}(\Gamma)$ and $\epsilon_{+}(\Gamma)$, and the bias of Eq. 11 as $\epsilon_{\mathrm{FSM}}$ respectively. Then the bias of FSM corresponding to the mask 
$\Gamma$ is calculated with piling up lemma:

$$
\epsilon(\Gamma)=2 \cdot \epsilon_{S^{\prime}}(\Gamma) \cdot 2^{3-1} \cdot \epsilon_{+}(\Gamma)^{3} .
$$

Now we describe how to search the 3 -tuples of mask values $\left(\Gamma, \Gamma \cdot \alpha, \Gamma \cdot \alpha^{-1}\right)$ which satisfies Eq. 13 and 14 with large bias. The procedure of the search for mask values is as follows:

Step 1. Make a list of mask values $\Gamma$ which satisfies Eq. 13 by using the linear profile of 8-bit permutation $S_{R}$ and the matrix $M$, where linear profile means the table of the biases of $S_{R}$ corresponding to all input and output masks.

Step 2. For each mask value $\Gamma$ acquired in Step 1, check if Eq. 13 holds for $\Gamma \cdot \alpha$ and $\Gamma \cdot \alpha^{-1}$ with large biases. We discard the candidate if one of biases associated with the 3 -tuple of masks is smaller than $2^{-20}$.

Step 3. Calculate the biases of Eq. 14 for the 3-tuples of masks acquired in Step 2.

Executing this procedure for all 32-bit mask values is impractical even after the restriction of the mask values. Especially evaluating the biases of Eq. 14 for all masks in detail by experiment requires much computational power. As a matter of fact, we examined $2^{12}$ random inputs for $x$ and $y$ in Step 3, but the error was too large to rely on the result.

Hence we restrict the domain of mask values of Eq. 14 in addition. Generally the bias of Eq. 14 tends to depend on the hamming weight of the mask value $\Gamma$, and tends to become large when the hamming weight is small.

Let the byte representation of the mask be denoted by $\Gamma=\left(\gamma_{3}, \gamma_{2}, \gamma_{1}, \gamma_{0}\right)$. We fixed the hamming weight of each $\gamma_{i}$ no more than two. The following lemma 1 and piling up lemma helps calculating the bias of 14 .

Lemma 1 Let us denote the probability of the incidence of carry at $n$-th bit from the least significant bit (LSB) as $p_{n}$.

1. If the hamming weight of $\Gamma$ is one (i.e. $\Gamma=0 \cdots 010 \cdots 0)$ and the $n$-th bit from the LSB is 1 , then the bias $\epsilon_{+}(\Gamma)$ is equal to $p_{n}-1 / 2$ :

$$
\epsilon_{+}(\Gamma)=p_{n}-1 / 2=2^{-n}-2^{-n-1} .
$$

2. If the mask value is $\Gamma=0 \cdots 0110 \cdots 0, \epsilon_{+}(\Gamma)=1 / 4$.

3 . If the mask value is $\Gamma=0 \cdots 01010 \cdots 0, \epsilon_{+}(\Gamma)=1 / 8$.

4. If the mask value is $\Gamma=0 \cdots 011110 \cdots 0, \epsilon_{+}(\Gamma)=1 / 8$.

\subsection{The Result}

Table 1 shows the best 3-tuples of approximation of FSM in our search.

The bias $\epsilon$ of the whole linear approximation Eq. 12 is estimated as follows:

$$
\epsilon=2^{4-1} \cdot \epsilon_{\mathrm{FSM}}(\Gamma)^{2} \cdot \epsilon_{\mathrm{FSM}}(\Gamma \cdot \alpha) \cdot \epsilon_{\mathrm{FSM}}\left(\Gamma \cdot \alpha^{-1}\right)=2^{-112.25} .
$$

As a result, about $2^{225}$ outputs $\left(2^{230}\right.$ bits $)$ are required to distinguish the output of SNOW 2.0 from a truly random bit sequence. 
Table 1. 3-tuple of masks of FSM and their biases

\begin{tabular}{l||l|l|l|l}
\hline & Mask value & $\epsilon_{S^{\prime}}(\cdot)$ & $\epsilon_{+}(\cdot)$ & $\epsilon_{\mathrm{FSM}}(\cdot)$ \\
\hline \hline$\Gamma$ & 0x0303600c & $2^{-15.61}$ & $2^{-5.00}$ & $2^{-27.61}$ \\
\hline$\Gamma \cdot \alpha$ & 0x0c030360 & $2^{-15.61}$ & $2^{-5.00}$ & $2^{-27.61}$ \\
\hline$\Gamma \cdot \alpha^{-1}$ & 0x03600c63 & $2^{-17.42}$ & $2^{-6.00}$ & $2^{-32.42}$ \\
\hline
\end{tabular}

\subsection{The Discussion}

Our evaluation confirmed that SNOW 2.0 is well improved. Especially inducing an MDS matrix in the 32-bit non-linear permutation $S^{\prime}$ reinforces the resistance property against linear masking attack. In fact, the original attack against SNOW 1.0 approximates the only one S-box to construct the best approximation of FSM. Contrary, four S-boxes become active in all approximation we found. This property is also important to the bias of Eq. 14 because the hamming weight of masks increases in general. As a result, the bias of the best approximation of FSM becomes quite small, from about $2^{-8}$ to $2^{-30}$.

On the other hand, the main reason this attack is applicable is caused by multiplying the primitive elements when we consider them as just a linear transformation over GF(2). This weakens the security of SNOW 2.0 against linear masking method. The choice of $\alpha$ or $\alpha^{-1}$ as the multiplier is effective in software implementation, but it weakens its security against linear masking attack. In fact, the mask $\Gamma$ is transformed by multiplying $\alpha$ and $\alpha^{-1}$ as follows:

$$
\begin{aligned}
\left(\gamma_{3}, \gamma_{2}, \gamma_{1}, \gamma_{0}\right) \alpha & =\left(\gamma^{\prime}, \gamma_{3}, \gamma_{2}, \gamma_{1}\right), \\
\left(\gamma_{3}, \gamma_{2}, \gamma_{1}, \gamma_{0}\right) \alpha^{-1} & =\left(\gamma_{2}, \gamma_{1}, \gamma_{0}, \gamma^{\prime \prime}\right) .
\end{aligned}
$$

Exactly one byte value is changed in each transformation.

Furthermore the bias associated with the mask $\Gamma=\left(0,0,0, \gamma^{\prime \prime}\right)$ is independent of the higher bits so that the bias associated with $\gamma^{\prime \prime}$ tends to be large even though the value is changeable and this bias is easy to calculate. Therefore if the bias $\epsilon_{+}(\Gamma)$ is large, the other two biases $\epsilon_{+}(\Gamma \cdot \alpha), \epsilon_{+}\left(\Gamma \cdot \alpha^{-1}\right)$ tend to be large, too.

\section{Conclusion}

In this paper we present the application of the linear (masking) attack proposed in [CHJ02b] to SNOW 2.0. The modified version seems to develop resistance to the original attack, though the most primitive technique is still applicable.

Our attack requires $2^{225}$ rounds outputs $\left(2^{230} \mathrm{bits}\right)$ and $2^{225}$ steps of analysis to distinguish the output of SNOW 2.0 from a truly random bit sequence. This concludes that SNOW 2.0 generates sufficiently random sequences as a 128-bit key stream cipher, but is slightly weak as a 256-bit key stream cipher. The fast correlation decoding technique could be applicable to extract a key by using this bias. We leave this problem for further research. 


\section{Acknowledgement}

We would like to thank Patrik Ekdahl. He pointed out that our experimental result in Table 1 was incorrect, and helped us to correct the values of the mask set and the corresponding biases.

\section{References}

[CHJ02a] D. Coppersmith, S. Halevi, and C. Jutla, "Scream: a software-efficient stream cipher," Fast Software Encryption, FSE 2002, LNCS 2365, pp. 195-209, 2002. 222

[CHJ02b] D. Coppersmith, S. Halevi, and C. Jutla, "Cryptanalysis of stream ciphers with linear masking," Advances in Cryptology, CRYPTO 2002, SpringerVerlag, LNCS 2442, pp. 515-532, 2002. 223, 225, 226, 231

[DC98] J. Daemen, C. Clapp, "Fast Hashing and Stream Encryption with Panama," Fast Software Encryption, FSE'98, Springer-Verlag, LNCS 1372, pp. 60-74, 1998. 222

[DR99] J. Daemen, V. Rijmen, "AES Proposal: Rijndael," AES algorithm submission, September 3, 1999, available at http://www.nist.gov/aes/.

[EJ00] P. Ekdahl and T. Johansson, "SNOW - a new stream cipher, " NESSIE project submission, 2000, available at http://www.cryptonessie.org. 222,224

[EJ02a] P. Ekdahl and T. Johansson, "Distinguishing attacks on SOBER-t16 and t32, " Fast Software Encryption, FSE 2002, Springer-Verlag, LNCS 2365, pp. 210-224, 2002. 223

[EJ02b] P. Ekdahl and T. Johansson, "A new version of the stream cipher SNOW, " Selected Areas in Cryptology, SAC 2002, Springer-Verlag, LNCS 2595, pp. 47-61, 2002. 222

[Go96] J. Golić, "Linear models for keystream generator," IEEE Trans. Computers, vol. C-45, pp. 41-49, 1996. 223

[Go97] J. Golić, "Linear statistical weakness of alleged RC4 keystream generator," Advances in Cryptology, Eurocrypt'97, Springer-Verlag, LNCS 1233, pp. 226-238. 223

[HR02] P. Hawkes and G. Rose, "Guess-and-Determine Attacks on SNOW," Selected Area of Cryptology, SAC 2002, Springer-Verlag, LNCS 2595, pp. 3746, 2002. 223

[Ma94] M. Matsui, "Linear cryptanalysis method for DES cipher," Advances in Cryptology, Proceedings Eurocrypt'93, Springer-Verlag, LNCS 765, pp. 159-169, 1994. 223, 225

[RC94] P. Rogaway, D. Coppersmith, "A Software-Optimized Encryption Algorithm," Fast Software Encryption, FSE'94, Springer-Verlag, LNCS 809, pp. 56-63, 1994. 222

[RC98] P. Rogaway, D. Coppersmith, "A Software-Optimized Encryption Algorithm," Journal of Cryptography, Vol. 11, No. 4, pp. 273-287, 1998. 222

[Ro98] G. Rose, "A Stream Cipher based on Linear Feedback over $G F\left(2^{8}\right)$," "Proc. Australian Conference on Information Security and Privacy," Springer-Verlag, 1998. 222

[Ru86] R. Rueppel, Analysis and Design of Stream Ciphers, Springer-Verlag, 1986. 222 
[WFYP02] D. Watanabe, S. Furuya, H. Yoshida, and B. Preneel, "A new keystream generator MUGI," Fast Software Encryption, FSE 2002, Springer-Verlag, LNCS 2365, pp. 179-194, 2002. 222 